H. Tamura

Nagoya Math. J.

Vol. 84 (1981), 169-193

\title{
THE PRINCIPLE OF LIMITING ABSORPTION FOR PROPAGATIVE SYSTEMS IN CRYSTAL OPTICS WITH PERTURBATIONS OF LONG-RANGE CLASS
}

\author{
HIDEO TAMURA
}

\section{§1. Introduction}

The present paper is a continuation of [10] where we have proved the principle of limiting absorption for uniformly propagative systems with perturbations of long-range class. In this paper, we consider the Maxwell equation in crystal optics as an important example of non-uniformly propagative systems and, under the same assumptions on perturbations as in [10], we prove the principle of limiting absorption for the stationary problem associated with this equation by using a way similar to that in [10]. We here restrict our consideration to a very special class of non-uniformly propagative systems, but the method developed in this paper will be applicable to more general systems for which non-zero roots of characteristic equations of unperturbed systems are at most double. For another works on the spectral and scattering problems for non-uniformly propagative systems with perturbations of short-range class, see [1], [5], [6], [7] and [8], etc.

1.1. Notations. We first list up the notations to be used throughout our entire discussion.

(1) We work exclusively in 3-dimensional euclidean space $R_{x}^{3}$ with generic point $x=\left(x_{1}, x_{2}, x_{3}\right) . \quad R_{\xi}^{3}$ denotes the 3 -dimensional space dual to $R_{x}^{3}$ and the generic point $\xi$ in $R_{\xi}^{3}$ is denoted by $\xi=\left(\xi_{1}, \xi_{2}, \xi_{3}\right)$. We further denote by $x \cdot \xi$ the scalar product between $x$ and $\xi ; x \cdot \xi=\sum_{j=1}^{3} x_{j} \xi_{j}$.

(2) $C^{k}$ denotes the $k$-dimensional unitary space with the usual scalar product (,). (In this paper, the notation (,) is used only for $k=6$.)

(3) For a multi-index $m=\left(m_{1}, m_{2}, m_{3}\right), m_{j}$ being a non-negative integer, we denote by $|m|$ the length of $m$. We write $\partial_{x}=\left(\partial / \partial x_{1}, \partial / \partial x_{2}, \partial / \partial x_{3}\right), D_{x}=$

Received March 5, 1980. 
$\left(D_{1}, D_{2}, D_{3}\right), D_{j}=-i \partial / \partial x_{j}(i=\sqrt{-1})$ and $\partial_{x}^{m}=\left(\partial / \partial x_{1}\right)^{m_{1}}\left(\partial / \partial x_{2}\right)^{m_{2}}\left(\partial / \partial x_{3}\right)^{m_{3}}$ for $m=\left(m_{1}, m_{2}, m_{3}\right)$. (We use the symbols $m$ and $n$ to denote multi-indices.)

1.2. Functional spaces. We introduce the functional spaces in which we work. We denote by $L_{2}$ the Hilbert space of square integrable functions over $R_{x}^{3}$ and introduce the space $L_{2, \alpha}$ with weight $\alpha$ by $L_{2, \alpha}=\{f$; $\left.\left(1+|x|^{2}\right)^{\alpha / 2} f \in L_{2}\right\}\left(L_{2,0}=L_{2}\right)$. We further define the space $L_{2, \alpha}^{(\ell)}$ as $L_{2, \alpha}^{(\ell)}=\Sigma$ $\oplus L_{2, \alpha}, \ell$ summands, and the norm in this space is denoted by ||$_{0, \alpha}^{(\ell)}$.

1.3. Formulation of results. We shall formulate the results to be obtained here with several assumptions. According to Courant-Hilbert [2], the propagative system of crystal optics in homogeneous media is described by

$$
(\nabla \times H)_{j}-\varepsilon_{j} \partial_{t} E_{j}=0, \quad(\nabla \times E)_{j}+\mu \partial_{t} H_{j}=0, \quad j=1,2,3,
$$

where $E={ }^{t}\left(E_{1}, E_{2}, E_{3}\right)$ and $H={ }^{t}\left(H_{1}, H_{2}, H_{3}\right)$ are the electric and magnetic field vectors, respectively, while $\varepsilon_{j}, \varepsilon_{j}>0$, and $\mu, \mu>0$, are the dielectric and magnetic permeability tensors, respectively. For simplicity, we assume throughout this paper that $\mu=1$ and that

$$
\varepsilon_{1}>\varepsilon_{2}>\varepsilon_{3} \text {. }
$$

(The case $\varepsilon_{1}=\varepsilon_{2}$ or $\varepsilon_{2}=\varepsilon_{3}$ can be dealt with similarly. In particular, when $\varepsilon_{1}=\varepsilon_{2}=\varepsilon_{3}$, equation (1.1) describes a uniformly propagative system in homogeneous isotropic media.)

Let $\Lambda\left(D_{x}\right)$ be the differential operator corresponding to $\nabla \times$;

$$
\Lambda(\xi)=\left(\begin{array}{rrr}
0, & -\xi_{3}, & \xi_{2} \\
\xi_{3}, & 0, & -\xi_{1} \\
-\xi_{2}, & \xi_{1}, & 0
\end{array}\right) .
$$

As is easily seen, $\Lambda(\xi)^{*}=-\Lambda(\xi), \Lambda(\xi)^{*}$ being the adjoint of $\Lambda(\xi)$. Equation (1.1) can be rewritten as a symmetric hyperbolic system for $u={ }^{t}\left(\varepsilon_{1}^{1 / 2} E_{1}\right.$, $\left.\varepsilon_{2}^{1 / 2} E_{2}, \varepsilon_{3}^{1 / 2} E_{3}, H_{1}, H_{2}, H_{3}\right)$

$$
-i \partial_{t} u=L_{0}\left(D_{x}\right) u
$$

where

$$
L_{0}(\xi)=\left(\begin{array}{cc}
0, & M_{0}^{-1 / 2} \Lambda(\xi) \\
-\Lambda(\xi) M_{0}^{-1 / 2}, & 0
\end{array}\right), \quad M_{0}=\left\{\varepsilon_{j} \delta_{j k}\right\}_{j, k=1,3},
$$

$\delta_{\jmath k}$ being Krocker's delta. 
The perturbed system associated with (1.3) which we investigate is given in the following form:

$$
-i \partial_{t} u=L\left(x, D_{x}\right) u
$$

where

$$
\begin{aligned}
& L(x, \xi)=\left(\begin{array}{cc}
0, & M(x)^{-1 / 2} \Lambda(\xi) N(x)^{-1 / 2} \\
-N(x)^{-1 / 2} \Lambda(\xi) M(x)^{-1 / 2}, & 0
\end{array}\right) . \\
& M(x)=\left\{\varepsilon_{j k}(x)\right\}_{j, k=1,3}, \quad N(x)=\left\{\mu_{j k}(x)\right\}_{j, k=1,3} .
\end{aligned}
$$

Here we make the following assumptions on $M(x)$ and $N(x)$ :

(A.1) $M(x)$ and $N(x)$ are positive definite uniformly in $x$;

(A.2) There exists a constant $\delta, \delta>0$, such that

$$
\begin{gathered}
\left|\varepsilon_{j k}(x)-\varepsilon_{j} \delta_{j k}\right| \leqq C(1+|x|)^{-\delta} \\
\left|\partial_{x}^{m} \varepsilon_{j k}(x)\right| \leqq C_{m}(1+|x|)^{-(1+|m| \delta)}, \quad|m| \geqq 1 ;
\end{gathered}
$$

(A.3) $\mu_{j k}(x)$ also has the same properties as above with $\varepsilon_{j} \delta_{j k}$ replaced by $\delta_{j k}$.

((1.8) seems to be rather restrictive, but we remark below that (1.8) with $|m|=1$ only is enough.)

Under the assumptions above, we see that the operator $L=L\left(x, D_{x}\right)$ defined by (1.6) has a natural self-adjoint realization (denoted by the same symbol $L)$ in $L_{2,0}^{(6)}$. The domain $\mathscr{D}(L)$ is given by $\mathscr{D}(L)=\left\{u ; u \in L_{2,0}^{(6)}, L u \in L_{2,0}^{(6)}\right\}$. Similarly, we denote by $L_{0}$ the self-adjoint realization of $L_{0}\left(D_{x}\right)$ defined by (1.4) with $\mathscr{D}\left(L_{0}\right)$.

We can now state the first result on the spectral properties of $L$. We always assume that (A.1) (A.3) are satisfied.

TheOREM 1.1. The eigenvalues of $L$ are discrete with possible accumulating points 0 and $\pm \infty$.

Next, we consider the following stationary equation associated with (1.5):

$$
L u-(\lambda \pm i \kappa) u=f, \quad 0<\kappa \leqq 1,
$$

with $f \in L_{2, \alpha}^{(6)}, \alpha>1 / 2, \lambda$ being the spectral parameter corresponding to the time variable $t$. Clearly, if $\kappa>0$, then there exists a unique solution $u=$ $R(\lambda \pm i \kappa) f=(L-(\lambda \pm i \kappa))^{-1} f$ such that $u \in L_{2, \alpha}^{(6)}$. The second result can be stated as follows: 
THEOREM 1.2. Assume that $\lambda, \lambda \neq 0$, is not an eigenvalue of $L$. Let $u=R(\lambda \pm i \kappa) f$ be the solution to equation (1.9) with $f \in L_{2, \alpha}^{(6)}, \alpha>1 / 2$. Then, the following statements hold:

(i) There exists a constant $C_{\alpha}$ independent of $\kappa, 0<\kappa \leqq 1$, such that

$$
|R(\lambda \pm i \kappa) f|_{0,-\alpha}^{(6)} \leqq C_{a}|f|_{0, \alpha}^{(6)} ;
$$

(ii) There exist bounded operators $R(\lambda \pm i 0)$ from $L_{2, \alpha}^{(6)}$ to $L_{2,-\alpha}^{(6)}$ defined by

$$
R(\lambda \pm i 0) f=\lim _{\kappa \downarrow 0} R(\lambda \pm i \kappa) f
$$

strongly in $L_{2,-\alpha}^{(6)}$.

1.4. Reduction. In order to prove the results above, we make the following reduction. By a simple transformation, equation (1.9) can be reduced to

$$
L_{0} u-(\lambda \pm i \kappa) u-(\lambda \pm i \kappa) E(x) u=f
$$

with another $f \in L_{2, \alpha}^{(6)}, \alpha>1 / 2$, ( $u$ being also another transformed function), where

$$
E(x)=\left(\begin{array}{cc}
M_{0}^{-1 / 2} M(x) M_{0}^{-1 / 2}, & 0 \\
0, & N(x)
\end{array}\right)-I_{6},
$$

$I_{6}$ being the $6 \times 6$ identity matrix. In view of (A.2) and (A.3), we see that each component of $E(x)$ converges to zero with order $|x|^{-\delta}$ as $|x| \rightarrow \infty$ and satisfies (1.8). To prove Theorem 1.2, it is sufficient to verify a similar result for solutions to equation (1.10).

1.5. Remark. We conclude this section by making some comments on assumption (A.2). As stated above, (1.8) in (A.2) is weakened. It will be easily seen that the argument used in the proof can be also applied to the case in which the perturbation $M(x)$ is decomposed into $M(x)=$ $M_{1}(x)+M_{2}(x)$, where $M_{1}(x)$ satisfies (A.2) and $M_{2}(x)$ is of short-range class (i.e. $M_{2}(x)$ satisfies (1.7) with $\left.\delta>1\right)$. Thus, for $M(x)$ satisfying (1.7) and (1.8) with $|m|=1$ only we define $M_{1}(x)$ as

$$
M_{1}(x)=\langle x\rangle^{-3 r} \int_{R^{s}} \rho\left((x-y)\langle x\rangle^{-r}\right) M(y) d y, \quad \delta>\gamma>0,
$$

$\left(\langle x\rangle=\left(1+|x|^{2}\right)^{1 / 2}\right)$ by use of the mollifier technique. Then, we see that $M_{1}(x)$ satisfies (1.8) with another $\delta(\delta=\gamma)$ and that $M_{1}(x)$ can be written in the above form. A similar decomposition is made for $N(x)$. 


\section{§2. Unperturbed propagative system}

In this section, we state several properties of the unperturbed system $L_{0}(\xi)$ (defined by (1.4)) which are necessary to the future argument.

2.1. Eigenvalues. According to [2] (pp. $602 \sim 607$ ), the eigenvalues of $L_{0}(\xi)$ are given as the roots of the equation

$$
\rho^{2}\left(\rho^{4}-\Psi(\xi) \rho^{2}+\Phi(\xi)\right)=0,
$$

where

$$
\begin{aligned}
& \Psi(\xi)=\varepsilon_{1}^{-1}\left(\xi_{2}^{2}+\xi_{3}^{2}\right)+\varepsilon_{2}^{-1}\left(\xi_{3}^{2}+\xi_{1}^{2}\right)+\varepsilon_{3}^{-1}\left(\xi_{1}^{2}+\xi_{2}^{2}\right), \\
& \Phi(\xi)=\left(\xi_{1}^{2}+\xi_{2}^{2}+\xi_{3}^{2}\right)\left\{\left(\varepsilon_{2} \varepsilon_{3}\right)^{-1} \xi_{1}^{2}+\left(\varepsilon_{3} \varepsilon_{1}\right)^{-1} \xi_{2}^{2}+\left(\varepsilon_{1} \varepsilon_{2}\right)^{-1} \xi_{3}^{2}\right\} .
\end{aligned}
$$

Hence, for $\xi \neq 0$, the matrix $L_{0}(\xi)$ has a zero eigenvalue with double multiplicity and four non-zero eigenvalues;

$$
\begin{aligned}
& \lambda_{ \pm 1}(\xi)= \pm \sqrt{\frac{1}{2}(\Psi(\xi)+\sqrt{X(\xi))}}, \\
& \lambda_{ \pm 2}(\xi)= \pm \sqrt{\frac{1}{2}(\Psi(\xi)-\sqrt{X(\xi))}}, \quad\left(\lambda_{j}=\lambda_{+j}, j=1,2\right)
\end{aligned}
$$

where

$$
\begin{aligned}
X(\xi) & =\Psi(\xi)^{2}-4 \Phi(\xi) \\
& =\left(\left(\sqrt{K_{1}} \xi_{1}-\sqrt{K_{3}} \xi_{3}\right)^{2}-K_{2} \xi_{2}^{2}\right)\left(\left(\sqrt{K_{1}} \xi_{1}+\sqrt{K_{3}} \xi_{3}\right)^{2}-K_{2} \xi_{2}^{2}\right) \geqq 0, \\
K_{1} & =1 / \varepsilon_{3}-1 / \varepsilon_{2}>0, \quad K_{2}=1 / \varepsilon_{1}-1 / \varepsilon_{3}<0, \quad K_{3}=1 / \varepsilon_{2}-1 / \varepsilon_{1}>0 .
\end{aligned}
$$

(The sign of $K_{j}(1 \leqq j \leqq 3)$ follows from (1.2).) Here we should note that when $\xi_{2}=0$ and $\sqrt{K_{1}} \xi_{1}-\sqrt{K_{3}} \xi_{3}=0$ (or $\left.\sqrt{K_{1}} \xi_{1}+\sqrt{K_{3}} \xi_{3}=0\right), \quad X(\xi)=0$. Therefore, $\lambda_{j}(\xi)(j=1,2)$ are not smooth at such points. Summing up, we have the following result.

LEMma 2.1. (i) The eigenvalues of $L_{0}(\xi), \xi \neq 0$, are enumerated so that

$$
\lambda_{1}(\xi) \geqq \lambda_{2}(\xi)>\lambda_{0}(\xi)=0>\lambda_{-2}(\xi) \geqq \lambda_{-1}(\xi)
$$

with relations $\lambda_{-j}(\xi)=-\lambda_{j}(\xi)$ and $\lambda_{j}(\xi)=\lambda_{j}(-\xi)(j=1,2)$.

(ii) $\lambda_{1}(\xi)=\lambda_{2}(\xi)$ at $\xi$ satisfying $X(\xi)=0$ and $\lambda_{1}(\xi)(j=1,2)$ are not smooth at such points.

(iii) $\lambda_{1}(\xi)+\lambda_{2}(\xi)=\sqrt{\Psi(\xi)+2 \sqrt{\Phi(\xi)}}, \lambda_{1}(\xi) \lambda_{2}(\xi)=\sqrt{\Phi(\xi)}$.

Hence, both of $\lambda_{1}(\xi)+\lambda_{2}(\xi)$ and $\lambda_{1}(\xi) \lambda_{2}(\xi)$ are smooth in $R_{\xi}^{3}-\{0\}$. 
(iv) $\operatorname{det}\left(L_{0}(\xi)-\lambda\right)=\lambda^{2} \prod_{j=1}^{2}\left(\lambda-\lambda_{j}(\xi)\right) \prod_{j=1}^{2}\left(\lambda-\lambda_{-j}(\xi)\right)$

$$
=\lambda^{2}\left(\lambda^{4}-\Psi(\xi) \lambda^{2}+\Phi(\xi)\right) \text {. }
$$

2.2. Slowness surface. From now on, we fix $\lambda$ so that $\lambda>0$ (the case $\lambda<0$ can be treated similarly).

We define the surfaces $\Xi_{j}(\lambda)(j=1,2)$ as

$$
E_{j}(\lambda)=\left\{\xi ; \lambda_{j}(\xi)=\lambda\right\} \text {. }
$$

Then, a short calculation shows that the two surfaces $\Xi_{1}(\lambda)$ and $\Xi_{2}(\lambda)$ intersect with each other at the following four points:

$$
\begin{aligned}
& p_{ \pm 1}=\left(\lambda \sqrt{\varepsilon_{3}\left(\varepsilon_{1}-\varepsilon_{2}\right) /\left(\varepsilon_{1}-\varepsilon_{3}\right)}, \quad 0, \quad \pm \lambda \sqrt{\left.\varepsilon_{1}\left(\varepsilon_{2}-\varepsilon_{3}\right) /\left(\varepsilon_{1}-\varepsilon_{3}\right)\right)},\right. \\
& p_{ \pm 2}=\left(-\lambda \sqrt{\varepsilon_{3}\left(\varepsilon_{1}-\varepsilon_{2}\right) /\left(\varepsilon_{1}-\varepsilon_{3}\right)}, 0, \pm \lambda \sqrt{\left.\varepsilon_{1}\left(\varepsilon_{2}-\varepsilon_{3}\right) /\left(\varepsilon_{1}-\varepsilon_{3}\right)\right)}\right. \text {. }
\end{aligned}
$$

LEMMA 2.2. (i) $\Xi_{j}(\lambda)(j=1,2), \lambda>0$, are bounded closed surfaces, enclosing the origin. (ii) $\Xi_{\jmath}(\lambda)$ are smooth except for the four points defined by (2.5).

2.3. Unitary matrix. From now on, we fix one of the four points defined by (2.5) and denote it by $\xi_{0}$. (For example, we take $p_{+1}$ as $\xi_{0}$.) Let $\mathcal{O}$ be a small neighborhood of $\xi_{0}$, not containing the origin. We denote by $\mathscr{E}_{ \pm}(\xi)$ and $\mathscr{E}_{0}(\xi), \xi \in \mathcal{O}$, the 2-dimensional subspaces spanned by the eigenvectors corresponding to $\lambda_{ \pm 1}(\xi)$ and $\lambda_{ \pm 2}(\xi)$ and to the zero eigenvalues, respectively. Then, we can find orthonormal bases $\left\{e_{+}^{j}(\xi)\right\}_{j=1,2}$ which span $\mathscr{E}_{+}(\xi)$ and which are smooth in $\mathcal{O}$. Furthermore, it is easily seen from definition that

$$
L_{0}\left(\xi_{0}\right) e_{+}^{j}\left(\xi_{0}\right)=\lambda_{i}\left(\xi_{0}\right) e_{+}^{j}\left(\xi_{0}\right), \quad j=1,2 \text {. }
$$

Similarly, we denote by $\left\{e_{-}^{j}(\xi)\right\}_{j=1,2}$ and $\left\{e_{0}^{j}(\xi)\right\}_{j=1,2}, \xi \in 0$, orthonormal bases spanning $\mathscr{E}_{-}(\xi)$ and $\mathscr{E}_{0}(\xi)$, respectively.

We define the $6 \times 6$ unitary matrix $U_{0}(\xi), \xi \in \mathcal{O}$, associated with the orthonormal bases above so that

$$
A_{0}(\xi)=U_{0}(\xi) L_{0}(\xi) U_{0}(\xi)^{*}=\left(\begin{array}{ccc}
A_{+}(\xi), & & \\
& 0, & \\
0 & & A_{-}(\xi)
\end{array}\right), \quad \xi \in \mathcal{O},
$$

where 


$$
\begin{aligned}
& A_{ \pm}(\xi)=\left(\begin{array}{ll}
a_{ \pm}^{11}(\xi), & a_{ \pm}^{12}(\xi) \\
a_{ \pm}^{21}(\xi), & a_{ \pm}^{22}(\xi)
\end{array}\right), \\
& a_{ \pm}^{j k}(\xi)=\left(L_{0}(\xi) e_{ \pm}^{k}(\xi), \quad e_{ \pm}^{j}(\xi)\right), \quad j, k=1,2 .
\end{aligned}
$$

Here we should note that $a_{ \pm}^{j k}(\xi)=0, j \neq k$, for $\xi$ satisfying $X(\xi)=0$. In particular, at $\xi=\xi_{0}$,

$$
a_{ \pm}^{11}\left(\xi_{0}\right)=a_{ \pm}^{22}\left(\xi_{0}\right)=\lambda_{ \pm 1}\left(\xi_{0}\right)=\lambda_{ \pm 2}\left(\xi_{0}\right)= \pm \lambda
$$

\section{§3. Weighted pseudo-differential operators}

In this section, we introduce a class of pseudo-differential operators and state several fundamental properties of these operators without proofs. Roughly speaking, the class to be defined here satisfies relations dual to the standard Hörmander class $S_{\rho, \delta}$ ([3]).

Definition 3.1. We say that $P(x, \xi)=\left\{p_{j k}(x, \xi)\right\}_{j, k=1, \ell},(x, \xi) \in R_{x}^{3} \times R_{\xi}^{3}$, belongs to $A_{\theta, \sigma}^{\beta, \gamma}(\ell)(1 \geqq \beta>\gamma \geqq 0, \sigma \geqq \theta+\gamma \geqq 0)$, when the following conditions are satisfied:

(a) $p_{j k}(x, \xi)$ is smooth in $R_{x}^{3} \times R_{\xi}^{3}$ and is rapidly decreasing in $\xi$ together with $x$-derivatives of all order;

(b) $\left|\partial_{\xi}^{n} p_{j k}(x, \xi)\right| \leqq C_{N, n}(1+|\xi|)^{-N}(1+|x|)^{-(\theta-|n| r)} \quad$ for any $N, N>0$;

(c) $\left|\partial_{x}^{m} \partial_{\xi}^{n} p_{j k}(x, \xi)\right| \leqq C_{N, m, n}(1+|\xi|)^{-N}(1+|x|)^{-\mu(m, n)},|m| \geqq 1$,

$$
(\mu(m, n)=\sigma+(|m|-1) \beta-|n| \gamma) .
$$

(The constants $\theta$ and $\sigma$ are decaying rates of $x$-derivatives of zero and first order, respectively.)

We say that a family of $P(x, \xi ; \varepsilon)$ with parameter $\varepsilon$ belongs to $A_{\theta, \sigma}^{\beta, r}(\ell)$ uniformly in $\varepsilon$, if the above constants $C_{N, n}$ and $C_{N, n, m}$ are taken independently of $\varepsilon$.

We now define the pseudo-differential operator $P=P\left(x, D_{x}\right)$ with symbol $P(x, \xi) \in A_{\theta, \sigma}^{\beta, r}(\ell)$ as follows:

$$
P u=(2 \pi)^{-3} \int e^{i x \cdot \xi} P(x, \xi) \hat{u}(\xi) d \xi
$$

for $u(x)={ }^{t}\left(u_{1}(x), \cdots, u_{\ell}(x)\right) \in \mathscr{S}, \mathscr{S}$ being the Schwartz space of rapidly decreasing smooth functions, where $\hat{u}(\xi)$ is the Fourier transform of $u(x)$; $\hat{u}(\xi)=\int e^{-i x \cdot \xi} u(x) d x$, and the integration with no domain attached is taken over the whole space.

Definition 3.2. We say that $P\left(x, D_{x}\right)$ belongs to $O P A_{\theta, o}^{\beta, r}(\ell)$, when it 
is a pseudo-differential operator with symbol $P(x, \xi) \in A_{\theta, \sigma}^{\beta, \gamma}(\ell)$.

In the future argument, we use frequently pseudo-differential operators with symbols having compact support in $\xi$.

The next properties are fundamental to pseudo-differential operators of class $O P A_{\theta, \sigma}^{\beta, \gamma}(\ell)$. The proof of these results can be done almost in the same way as in the Hörmander class $S_{\rho, \delta}$, so we omit it ([3], [4]).

Proposition 3.1. (i) Let $P_{j}\left(x, D_{x}\right)(j=1,2)$ be pseudo-differential operators of class $O P A_{\theta_{j}, \sigma_{j}}^{\beta, r}(\ell)$. Then, the product $P=P_{1} P_{2}$ is also a pseudodifferential operator of class $O P A_{\theta, \sigma}^{\beta, \gamma}(\ell)$, where $\theta=\theta_{1}+\theta_{2}$ and $\sigma=\min \left(\sigma_{1}+\theta_{2}\right.$, $\left.\sigma_{2}+\theta_{1}\right)$, and the symbol $P(x, \xi)$ is expressed as $P(x, \xi)=P_{1}(x, \xi) P_{2}(x, \xi)+$ $Q(x, \xi)$ with $Q(x, \xi) \in A_{\rho, \sigma}^{\beta, r}(\ell), \rho=\sigma-\gamma$.

(ii) Let $P\left(x, D_{x}\right)$ be of class $O P A_{\theta, \sigma}^{\beta, \gamma}(\ell)$. Then, $P^{*}, P^{*}$ being the adjoint of $P$ in $L_{2,0}^{(\ell)}$, is also a pseudo-differential operator of class $O P A_{\theta, \sigma}^{\beta, \gamma}(\ell)$ and the symbol $\sigma\left(P^{*}\right)(x, \xi)$ is expressed as $\sigma\left(P^{*}\right)(x, \xi)=P^{*}(x, \xi)+Q(x, \xi)$ with $Q(x, \xi) \in A_{\rho, \sigma}^{\beta, \gamma}(\ell), \rho=\sigma-\gamma$, where $P^{*}(x, \xi)$ is the adjoint matrix of $P(x, \xi)$.

Proposition 3.2. Let $P\left(x, D_{x}\right)$ be of class $O P A_{\theta, \sigma}^{\beta, r}(\ell)$. Then, $P$ is a bounded operator from $L_{2, \alpha}^{(\ell)}$ to $L_{2, \alpha+\theta}^{(\ell)}$ for any $\alpha$. Furthermore, if $P\left(x, D_{x} ; \varepsilon\right)$ belongs to $O P A_{\theta, \sigma}^{\beta, \gamma}(\ell)$ uniformly in $\varepsilon$, then $P\left(x, D_{x} ; \varepsilon\right)$ is bounded uniformly.

\section{§4. Diagonalization}

4.1. Decomposition. We fix $\theta, 0<\theta<1$, so that $\theta<\delta$ for $\delta$ in (A.2). Let $E(x)$ be the symmetric matrix defined by (1.11) and let $e_{j k}(x)(j, k=$ $1,6)$ be each component of $E(x)$. Then, we can make the following decomposition: For any $\varepsilon>0$ small enough, there exists a constant $R=$ $R(\theta, \varepsilon)$ such that; (i) $e_{j k}(x)=e_{j k}(x ; \varepsilon)+\tilde{e}_{j k}(x ; \varepsilon)$, (ii) $e_{j k}(x ; \varepsilon)=e_{j k}(x)$ for $|x|$ $\geqq R$ and hence $\tilde{e}_{j k}(x ; \varepsilon)$ is of compact support, (iii) $\left|e_{j k}(x ; \varepsilon)\right| \leqq \varepsilon(1+|x|)^{-\theta}$ and $\left|\partial_{x}^{m} e_{j k}(x ; \varepsilon)\right| \leqq \varepsilon C_{m}(1+|x|)^{-(1+|m| \theta)}$ for all $x$. We denote by $E(x ; \varepsilon)$ the symmetric matrix with components $e_{j k}(x ; \varepsilon) ; E(x ; \varepsilon)=\left\{e_{j k}(x ; \varepsilon)\right\}_{j, k=1,6}$.

We consider the following equation (see (1.10)):

$$
L_{0} u-(\lambda+i \kappa) u-(\lambda+i \kappa) E(x ; \varepsilon) u=f, \quad 0<\kappa \leqq 1,
$$

with $f \in L_{2, \alpha}^{(6)}, \alpha>\frac{1}{2}$. Our aim of this and the next sections is to reduce (4.1) to an equation of the form like (3.1) in [10] through several steps. To do this, we treat for the moment the equation (4.1) with $\kappa=0$;

$$
L_{0} u-\lambda u-\lambda E(x ; \varepsilon) u=f
$$


and assume that the solution $u$ of (4.2) belongs to $L_{2,0}^{(8)}$.

From now on, we fix the constant $\theta$ with the meaning ascribed here and set $\sigma=1+\theta$ ( $\sigma$ is also fixed).

4.2. Localization. Let $\xi_{0}$ be the point fixed in subsection $2.3\left(\xi_{0}=p_{+1}\right)$. Let $\mathcal{O}$ be a small neighborhood of $\xi_{0}$, not containing the origin. (We fix $\mathcal{O}$ throughout the remainder.)

Let $\psi(\xi)$ be a non-negative smooth function supported in $\mathcal{O}$ such that $\psi(\xi)=1$ in a small neighborhood of $\xi_{0}$. We let $\psi\left(D_{x}\right)$ operate on both sides of equation (4.2) to obtain

$$
\left(L_{0}-\lambda-\lambda E(x ; \varepsilon)\right) \psi\left(D_{x}\right) u=\psi\left(D_{x}\right) f+r(x ; \varepsilon),
$$

where $r(x ; \varepsilon)=\lambda\left[\psi\left(D_{x}\right), E(x ; \varepsilon)\right] u,[$,$] being the commutator. By decom-$ position in 4.1 and by Proposition 3.1, the pseudo-differential operator $\varepsilon^{-1} \lambda\left[\psi\left(D_{x}\right), E(x ; \varepsilon)\right]$ belongs to $O P A_{\sigma, o}^{\theta, 0}(6)$ uniformly in $\varepsilon$. Here we introduce the following notation.

Notation 4.1. We denote by $r(\varepsilon)=r(x ; \varepsilon)$ all terms which are written as $r(\varepsilon)=R\left(x, D_{x} ; \varepsilon\right) u$ for the solution $u$ of (4.2) with $R\left(x, D_{x} ; \varepsilon\right) \in O P A_{\sigma, \sigma}^{\theta, 0}(6)$ such that the symbol $\varepsilon^{-1} R(x, \xi ; \varepsilon)$ belongs to $A_{\sigma, \sigma}^{\theta, 0}(6)$ uniformly in $\varepsilon$.

According to Proposition 3.2, we see that

$$
|r(\varepsilon)|_{0, \nu+\sigma}^{(6)} \leqq \varepsilon C|u|_{0, \nu}^{(6)} \quad \text { for any } \nu,
$$

if $u \in L_{2, \nu^{*}}^{(6)}$

4.3. Diagonalization. The next task is to transform (4.3) into an equation of the diagonalized form. This transformation is made on the basis of the lemma below (Lemma 4.1). To formulate this lemma, it is convenient to introduce the following definition and notation.

Definition 4.1. We say that $P(x, \xi)$ defined on $R_{x}^{3} \times \mathcal{O}$ belongs to $A_{\mu, \nu}^{\beta, \gamma}(\ell ; \mathcal{O})$, if $\phi(\xi) P(x, \xi) \in A_{\mu, \nu}^{\beta, \gamma}(\ell)$ for any smooth function $\phi(\xi)$ supported in $\mathcal{O}$.

Notation 4.2. For a vector-valued function $v$ with 6-components, we write $v={ }^{t}\left(v_{+}(x), v_{0}(x), v_{-}(x)\right)$, where $v_{ \pm}(x)={ }^{t}\left(v_{ \pm 1}(x), v_{ \pm 2}(x)\right)$ and $v_{0}(x)={ }^{t}\left(v_{01}(x)\right.$, $v_{02}(x)$ ). Roughly speaking, $v_{ \pm}$and $v_{0}$ correspond to the spaces $\mathscr{E}_{ \pm}$and $\mathscr{E}_{0}$ introduced in subsection 2.3, respectively. For given function $v$, we often write $v_{ \pm}=[v]_{ \pm}$and $v_{0}=[v]_{0}$ to denote the \pm -components and 0-components, respectively. 
LEMma 4.1. Let $U_{0}(\xi), \xi \in \mathcal{O}$, be the unitary matrix introduced in (2.7) and let $A_{0}(\xi)$ be defined by (2.7). Set $L(\varepsilon)=L(x, \xi ; \lambda, \varepsilon)=L(x, \xi)-\lambda-$ $\lambda E(x ; \varepsilon),(x, \xi) \in R_{x}^{3} \times \mathcal{O}$, for $L(x, \xi)$ defined by (1.6). Then, for any $\varepsilon>0$ small enough, there exists a $6 \times 6$ matrix $U(\varepsilon)=U(x, \xi ; \lambda, \varepsilon)$ such that

$$
U(\varepsilon) L(\varepsilon) U(\varepsilon)^{-1}=A_{0}(\xi)-\lambda+X(\varepsilon)+R(\varepsilon)
$$

in $R_{x}^{3} \times \mathcal{O}$. Here $U(\varepsilon), X(\varepsilon)=X(x, \xi ; \lambda, \varepsilon)$ and $R(\varepsilon)=R(x, \xi ; \lambda, \varepsilon)$ have the following properties:

(a) $R(\varepsilon)$ belongs to $A_{\sigma, \sigma}^{\theta, 0}(6 ; \mathcal{O})$;

(b) $X(\varepsilon)$ is of the following form:

$$
X(\varepsilon)=\left(\begin{array}{ccc}
X_{+}(\varepsilon), & & \\
& 0, & \\
0 & & \\
& & X_{-}(\varepsilon)
\end{array}\right),
$$

where $X_{ \pm}(\varepsilon)$ and $X_{0}(\varepsilon)$ are $2 \times 2$ symmetric matrices and belong to $A_{\theta, o}^{\theta, 0}(2 ; \mathcal{O})$;

(c) $U(\varepsilon)$ is represented in the form

$$
U(\varepsilon)=U_{0}(\xi)+U_{1}(x, \xi ; \lambda, \varepsilon)
$$

with $U_{1}(\varepsilon) \in A_{\theta, \sigma}^{\theta, 0}(6 ; \mathcal{O})$. Furthermore, $U(\varepsilon)$ satisfies

$$
U(\varepsilon) U(\varepsilon)^{*}=I_{6}-R_{1}(x, \xi ; \lambda, \varepsilon)
$$

with $R_{1}(\varepsilon) \in A_{\sigma, o}^{\theta, 0}(6 ; \mathcal{O}), I_{6}$ being the $6 \times 6$ identity matrix.

(d) $\varepsilon^{-1} R(\varepsilon), \varepsilon^{-1} X_{ \pm}(\varepsilon), \varepsilon^{-1} X_{0}(\varepsilon), \varepsilon^{-} U_{1}(\varepsilon)$ and $\varepsilon^{-1} R_{1}(\varepsilon)$ belong to the corresponding symbol classes uniformly in $\varepsilon$.

We have proved a result similar to Lemma 4.1 in Appendix of [9], where $A_{0}(\xi)$ was assumed to be diagonal. However, this lemma can be also verified with a slight modification in exactly the same way as in [9], so we omit the proof.

We now transform (4.3) into an equation of the diagonalized form. Let $\chi(\xi)$ be a non-negative smooth function supported in $\mathcal{O}$ such that $\chi(\xi) \psi(\xi)$ $=\psi(\xi)$ for $\psi(\xi)$ introduced at the beginning of subsection 4.2. We define $\tilde{U}(x, \xi ; \lambda, \varepsilon) \in A_{0, \sigma}^{\theta, 0}(6)$ as $\tilde{U}(x, \xi ; \lambda, \varepsilon)=\chi(\varepsilon) U(\varepsilon)$ with $U(\varepsilon)$ in Lemma 4.1. Similarly, we define $\tilde{A}_{0}(\xi), \tilde{X}_{ \pm}(\varepsilon)$ and $\tilde{X}_{0}(\varepsilon)$. We set

$$
v=\tilde{U}\left(x, D_{x} ; \lambda, \varepsilon\right) \psi\left(D_{x}\right) u .
$$

Then, we have the next result. 
Lemma 4.2. Let $\tilde{X}_{+}(\varepsilon)$ be as above and let $v$ be defined by (4.4). Then, $v_{+}=[v]_{+}$satisfies the equation

$$
\left(\tilde{A}_{+}\left(D_{x}\right)-\lambda I_{2}+\tilde{X}_{+}\left(x, D_{x} ; \lambda, \varepsilon\right)\right) v_{+}=g+[r(\varepsilon)]_{+}
$$

with some $r(\varepsilon)$, where $\tilde{A}_{+}(\xi)$ is defined by $\tilde{A}_{+}(\xi)=\chi(\xi) A_{+}(\xi)$ with $A_{+}(\xi)$ given by (2.8) and $I_{2}$ is the $2 \times 2$ identity matrix, while $g$ is defined by

$$
g=\left[\tilde{U}\left(x, D_{x} ; \lambda, \varepsilon\right) \psi\left(D_{x}\right) f\right]_{+} .
$$

$v_{-}\left(\right.$resp. $\left.v_{0}\right)$ satisfies a similar equation with $\tilde{A}_{+}\left(D_{x}\right)$ and $\tilde{X}_{+}(\varepsilon)$ replaced by $\tilde{A}_{-}\left(D_{x}\right)$ and $\tilde{X}_{-}(\varepsilon)$ (resp. 0 and $\tilde{X}_{0}(\varepsilon)$ ).

The proof of this lemma is done by using Lemma 4.1 and by making an easy calculation based on Propositions 3.1 and 3.2.

Here we should note that the equations for $v_{-}$and $v_{0}$ can be easily treated, since the symbols of pseudo-differential operators in these equations are invertible in a neighborhood of the support of $\psi(\xi)$ for $\varepsilon$ small enough. Thus, we consider the equation only for $v_{+}$in the next sections.

\section{§5. Transformation, I}

In this and the next sections, we consider the equation (4.5) and transform this into an equation of the desired form ((3.1) in [10]) through two steps.

5.1. Change of the coordinates. We first introduce the new coordinates $(\tau, \eta)$ in $R_{\xi}^{3}$ as follows:

$$
\tau=\left(\sqrt{K_{3}} \xi_{1}+\sqrt{K_{1}} \xi_{3}\right) / \sqrt{K}, \quad \eta_{1}=\left(\sqrt{K_{1}} \xi_{1}-\sqrt{K_{3}} \xi_{3}\right) / \sqrt{K}, \quad \eta_{2}=\xi_{2},
$$

where $K_{j}(1 \leqq j \leqq 3)$ are the constants defined in subsection 2.1 and $K=$ $K_{1}+K_{3}$. We write $\zeta=(\tau, \eta), \eta=\left(\eta_{1}, \eta_{2}\right)$. Then, there exists a constant unitary matrix $\Pi$ of size $3 \times 3$ such that $\zeta=\Pi \xi$. We further denote by $z=(t, y), y=\left(y_{1}, y_{2}\right)$, the coordinates (in $R_{x}^{3}$ ) dual to $\zeta ; z=\Pi^{*} x$. The unitary matrix $\Pi$ induces naturally the one to one map (denoted by the same symbol $\Pi) ;(\Pi \phi)(z, \zeta)=\phi\left(\Pi z, \Pi^{*} \zeta\right)$. For notational convenience, we denote a representation in terms of the $(z, \zeta)$ coordinates by the same symbol as an original function which is represented in terms of the $(x, \xi)$ coordinates; $\phi(z, \zeta)=(\Pi \phi)(z, \zeta)=\phi\left(\Pi z, \Pi^{*} \zeta\right)$ for $\phi=\phi(x, \xi)$. Clearly, $\Pi$ is a unitary map from $L_{2,0}^{(l)}$ to itself and the symbol class $A_{\nu, \mu}^{\beta, r}(\ell)$ defined in section 3 is stable under this transformation. 
5.2. Preparations. Let $\xi_{0}$ be the point fixed in subsection $2.3\left(\xi_{0}=p_{+1}\right)$. Then, $\xi_{0}$ is represented in the $\zeta$-coordinates as follows: $\xi_{0}=\left(\tau_{0}, 0,0\right)$. (The explicit expression for $\tau_{0}$ is not needed later.) We take the (fixed) small neighborhood $\mathcal{O}$ of $\xi_{0}$ in the form $\mathcal{O}=J \times \Omega$, where $J$ is a small open interval containing $\tau_{0}$ and $\Omega\left(\subset R_{\eta}^{2}\right)$ is a small neighborhood of the origin $(0,0)$.

We state several simple facts which will be used in the later argument. Let $\Psi(\xi)(=\Psi(\zeta))$ and $\Phi(\xi)(=\Phi(\zeta))$ be defined by (2.2) and (2.3), respectively. Then, we have

$$
\lambda^{4}-\Psi(\xi) \lambda^{2}+\Phi(\xi)=C\left(\tau^{4}+\sum_{j=0}^{3} \Gamma_{j}(\eta ; \lambda) \tau^{3-j}\right), \quad \zeta \in \mathcal{O},
$$

with some coefficients $\Gamma_{j}(\eta ; \lambda)(0 \leqq j \leqq 3)$ smooth in $\Omega, C$ being a positive constant. Furthermore, the right side of (5.2) is decomposed as

$$
\begin{aligned}
\tau^{4}+ & \sum_{j=0}^{3} \Gamma_{\jmath}(\eta ; \lambda) \tau^{3-j} \\
& =\left(\tau^{2}-\sigma_{1}(\eta ; \lambda) \tau+\sigma_{2}(\eta ; \lambda)\right)\left(\tau^{2}+\tilde{\sigma}_{1}(\eta ; \lambda) \tau+\tilde{\sigma}_{2}(\eta ; \lambda)\right),
\end{aligned}
$$

where all the coefficients $\sigma_{j}(\eta ; \lambda)$ and $\tilde{\sigma}_{j}(\eta ; \lambda)(j=1,2)$ are positive and smooth in $\Omega$. Let $\tau_{j}(\eta ; \lambda)(j=1,2), \tau_{1} \leqq \tau_{2}$, be the (positive) roots of the equation

$$
\tau^{2}-\sigma_{1}(\eta ; \lambda) \tau+\sigma_{2}(\eta ; \lambda)=0 .
$$

Then, the surfaces defined by $\lambda_{j}(\xi)=\lambda(j=1,2)$ are represented as $\tau=$ $\tau_{j}(\eta ; \lambda)$ in the $(\tau, \eta)$ coordinates. Furthermore, using (iv) in Lemma 2.1 and recalling the definition of $A_{+}(\xi)$, we have

$$
\begin{aligned}
\operatorname{det}\left(A_{+}(\xi)-\lambda\right) & =\left(\lambda-\lambda_{1}(\xi)\right)\left(\lambda-\lambda_{2}(\xi)\right) \\
& =D(\tau, \eta ; \lambda)\left(\tau^{2}-\sigma_{1}(\eta ; \lambda) \tau+\sigma_{2}(\eta ; \lambda)\right), \quad \zeta \in \mathcal{O},
\end{aligned}
$$

where

$$
D(\tau, \eta ; \lambda)=\frac{C\left(\tau^{2}+\tilde{\sigma}_{1}(\eta ; \lambda) \tau+\tilde{\sigma}_{2}(\eta ; \lambda)\right)}{\left(\lambda+\lambda_{1}(\xi)\right)\left(\lambda+\lambda_{2}(\xi)\right)}
$$

$C$ being as in (5.2). Clearly, $D(\tau, \eta ; \lambda)$ is positive and smooth in $\mathcal{O}$.

LEMMA 5.1. Let $A_{+}(\xi)\left(=A_{+}(\zeta)\right), \zeta \in \mathcal{O}$, be the $2 \times 2$ symmetric matrix defined by (2.8) and let $a_{+}^{j k}(\xi)\left(=a_{+}^{j k}(\zeta)=a_{+}^{j k}(\tau, \eta)\right)(j, k=1,2)$ be the $(j, k)$ component of $A_{+}(\xi)$. Let $\lambda_{j}(\xi)\left(=\lambda_{j}(\tau, \eta)\right)$ be the positive eigenvalues of $L_{0}(\xi)$ 
(defined by (2.4)). Let $\sigma_{j}(\eta ; \lambda)(j=1,2)$ be as above. Then, the following facts hold:

(i) $a_{+}^{11}\left(\tau_{0}, 0\right)+a_{+}^{22}\left(\tau_{0}, 0\right)=2 \lambda$ at $\xi_{0}=\left(\tau_{0}, 0,0\right)$;

(ii) $a_{+}^{12}(\tau, 0)=a_{+}^{21}(\tau, 0)=0$ for $\tau \in J$;

(iii) $(\partial / \partial \tau)\left(\lambda_{1}\left(\tau_{0}, 0\right)+\lambda_{2}\left(\tau_{0}, 0\right)\right)>0$;

(iv) $\sigma_{1}(0 ; \lambda)=2 \tau_{0}$.

Proof. Since $\lambda_{j}\left(\xi_{0}\right)=\lambda(j=1,2)$ by definition, (i) follows from (2.9) at once. By our choice of the $(\tau, \eta)$ coordinates, we easily see that $\lambda_{1}(\tau, 0)$ $=\lambda_{2}(\tau, 0)$ for $\tau \in J$. Indeed, $X(\xi)=0$ at such points, $X(\xi)$ being as in (2.4). Hence, the above fact follows immediately and (ii) is also verified. Since $\lambda_{j}(\xi)(j=1,2)$ are positively homogeneous of degree one and since $\tau$ is a vector transversal to the surfaces defined by $\lambda_{j}(\xi)=\lambda$ (not smooth) at $\xi=\xi_{0}$, (iii) is easily verified. By a simple geometric consideration, we see that the point $\left(2 \tau_{0}, 0,0\right)$ lies on the surface defined by $\tau=\sigma_{1}(\eta ; \lambda)$ and hence (iv) follows at once.

5.3. The first step. The first step of transformation is based on the following lemma.

Lemma 5.2. Let $A_{+}(\xi)\left(=A_{+}(\zeta)\right), \zeta \in \mathcal{O}$, be defined by (2.8). Then, there exist two $2 \times 2$ matrices $S(\zeta)$ and $B(\eta)$ such that

$$
S(\zeta)\left(A_{+}(\zeta)-\lambda I_{2}\right) S(\zeta)=\tau I_{2}-B(\eta), \quad \zeta \in \mathcal{O},
$$

$I_{2}$ being the $2 \times 2$ identity matrix. Here $S(\zeta)$ and $B(\eta)$ have the following properties:

(a) $S(\zeta)$ is symmetric, invertible and smooth in $\mathcal{O}$;

(b) $B(\eta)$ is symmetric and smooth in $\Omega, \mathcal{O}=J \times \Omega$.

The proof of this lemma is rather long, so we give it in section 7 . We admit the validity of Lemma 5.2 for the moment and continue the transformation.

Let $\chi(\xi)(=\chi(\zeta))$ be the function introduced in subsection 4.3. Let $\omega(\eta)$ be a non-negative smooth function supported in $\Omega$ such that $\omega(\eta) \chi(\zeta)=$ $\chi(\zeta)$. We define the symbols $\tilde{S}(\zeta), \tilde{V}(\zeta)$ and $\tilde{B}(\eta)$ as $\tilde{S}(\zeta)=\chi(\zeta) S(\zeta), \tilde{V}(\zeta)=$ $\chi(\zeta) S(\zeta)^{-1}$ and $\tilde{B}(\eta)=\omega(\eta) B(\eta)$, respectively, with $S(\zeta)$ and $B(\eta)$ introduced in Lemma 5.2. We further define $\tilde{Y}_{+}(z, \zeta ; \lambda, \varepsilon)$ as

$$
\tilde{Y}_{+}(z, \zeta ; \lambda, \varepsilon)=\tilde{S}(\zeta) \tilde{X}_{+}(z, \zeta ; \lambda, \varepsilon) \tilde{S}(\zeta)
$$

for $\tilde{X}_{+}(z, \zeta ; \lambda, \varepsilon)=\tilde{X}_{+}(x, \xi ; \lambda, \varepsilon)$ in equation (4.5). Here it should be noted 
that $\tilde{Y}_{+}(z, \zeta ; \lambda, \varepsilon)$ is symmetric and belongs to $A_{\theta, \sigma}^{\theta, 0}(2)$. We set

$$
\tilde{v}_{+}=\tilde{V}\left(D_{z}\right) v_{+}=\tilde{V}\left(D_{z}\right)\left[\tilde{U}\left(z, D_{z} ; \lambda, \varepsilon\right) \psi\left(D_{z}\right) u\right]_{+}
$$

for the solution $v_{+}$of equation (4.5). Then, we have the following result.

LEMMA 5.3. Let the symbols $\tilde{S}(\zeta), \tilde{B}(\eta)$ and $\tilde{Y}_{+}(z, \zeta ; \lambda, \varepsilon)$ be as above. Let $\tilde{v}_{+}$be defined by (5.9). Then, $\tilde{v}_{+}$obeys the equation

$$
\left(D_{t}-\tilde{B}\left(D_{y}\right)+\tilde{Y}_{+}\left(z, D_{z} ; \lambda, \varepsilon\right)\right) \tilde{v}_{+}=\tilde{S}\left(D_{z}\right) g+[r(\varepsilon)]_{+}
$$

with some $r(\varepsilon)$, where $g$ is defined by (4.6).

Equation (5.10) is easily derived by letting $\tilde{S}\left(D_{z}\right)$ operate on both sides of (4.5) and by making use of Lemma 5.2. This is the equation obtained through the first step.

\section{§6. Transformation, II}

6.1. Symbol class. To formulate the results obtained in this section, it is convenient to introduce the new symbol class which is a subclass of $A_{\nu, \mu}^{\beta, r}(\ell)$ defined in section 3.

Definition 6.1. We say that $P(z, \zeta)=\left\{p_{j k}(z, \zeta)_{j, k=1, \ell}, \zeta=(\tau, \eta)\right.$, belongs to $B_{\nu, \mu}^{\beta, \gamma}(\ell)(\mu \geqq \nu+\gamma \geqq 0,1 \geqq \beta>\gamma \geqq 0)$, if the following conditions are satisfied:

(a) $P(z, \zeta) \in A_{\nu, \mu}^{\beta, \gamma}(\ell)$;

(b) $\left|\partial_{\tau}^{i} \partial_{\eta}^{n} p_{j k}(z, \zeta)\right| \leqq C_{0}(1+|\zeta|)^{-N}(1+|z|)^{-(\nu-|n| r)}$ for any $N$;

(c) $\left|\partial_{z}^{m} \partial_{z}^{i} \partial_{\eta}^{n} p_{j k}(z, \zeta)\right| \leqq C_{m}(1+|\zeta|)^{-N}(1+|z|)^{-\mu(m, n)},|m| \geqq 1$,

$$
(\mu(m, n)=\mu+(|m|-1) \beta-|n| \gamma)
$$

where the constants $C_{0}$ and $C_{m}$ may depend on $i, n$ and $N$.

We further define $B_{\nu, \mu}^{\beta, \gamma}(\ell ; \mathcal{O}), \mathcal{O}=J \times \Omega$, in the same way as $A_{\nu, \mu}^{\beta, \gamma}(\ell ; \mathcal{O})$ was defined (Definition 4.1). In particular, we denote by $\tilde{B}_{\nu, \mu}^{\beta, r}(\ell ; \Omega)$ the subclass of $B_{\nu, \mu}^{\beta, r}(\ell ; \mathcal{O})$ such that $P(z, \zeta)=P(z, \tau, \eta)$ is independent of $\tau, \tau \in J$; $P(z, \zeta)=P(z, \eta)$.

6.2. The second step. We fix $\gamma$ so that $0<\gamma<\theta$ and set $\rho=\sigma-\gamma$ $=1+\theta-\gamma>1$. (From now on, we use the constants $\gamma$ and $\rho$ with the meanings ascribed here.) The second step is based on the following lemma.

LEMMA 6.1. Let $B(\eta), \eta \in \Omega$, be as in Lemma 5.2 and let $\tilde{Y}_{+}(\varepsilon)=\tilde{Y}_{+}(z, \zeta$; $\lambda, \varepsilon) \in A_{\theta, \sigma}^{\theta, 0}(2)$ be defined by (5.8). Then, there exist two $2 \times 2$ matrices $P(\varepsilon)$ $=P(z, \zeta ; \lambda, \varepsilon)$ and $Q(\varepsilon)=Q(z, \eta ; \lambda, \varepsilon)$ such that 


$$
\begin{aligned}
\left(I_{2}+\right. & P(\varepsilon))\left(\tau I_{2}-B(\eta)+Y_{+}(\varepsilon)\right)\left(I_{2}+P(\varepsilon)\right) \\
& =\tau I_{2}-B(\eta)+Q(\varepsilon)+R_{2}(\varepsilon), \quad \zeta \in \mathcal{O},
\end{aligned}
$$

with some $R_{2}(\varepsilon)=R_{2}(z, \zeta ; \lambda, \varepsilon) \in B_{o, \sigma}^{\theta, r}(2 ; \mathcal{O})$. Here $P(\varepsilon), Q(\varepsilon)$ and $R_{2}(\varepsilon)$ have the following properties:

(a) $P(\varepsilon)$ is symmetric and belongs to $B_{\theta, \sigma}^{\theta, r}(2 ; \mathcal{O})$. Furthermore, $I_{2}+P(\varepsilon)$ is invertible in $\mathcal{O}$ for $\varepsilon$ small enough.

(b) $Q(\varepsilon)$ is symmetric and belongs to $\tilde{B}_{\theta, r}^{\theta, r}(2 ; \Omega)$.

(c) $\varepsilon^{-1} R_{2}(\varepsilon), \varepsilon^{-1} P(\varepsilon)$ and $\varepsilon^{-1} Q(\varepsilon)$ belong to the corresponding symbol classes uniformly in $\varepsilon$.

The proof of this lemma is rather long, so we give it in section 8 . We admit that Lemma 6.1 is valid and proceed to the transformation.

Let $\chi(\zeta)$ and $\omega(\eta)$ be as before. We define the symbols $\tilde{T}(\varepsilon)=\tilde{T}(z, \zeta ; \lambda, \varepsilon)$ and $\tilde{W}(\varepsilon)=\tilde{W}(z, \zeta ; \lambda, \varepsilon)$ as

$$
\tilde{T}(\varepsilon)=\chi(\zeta)\left(I_{2}+P(z, \zeta ; \lambda, \varepsilon)\right), \tilde{W}(\varepsilon)=\chi(\zeta)\left(I_{2}+P(z, \zeta ; \lambda, \varepsilon)\right)^{-1}
$$

with $P(z, \zeta ; \lambda, \varepsilon)$ introduced in Lemma 6.1. Clearly, $\tilde{T}(\varepsilon)$ and $\tilde{W}(\varepsilon)$ belong to $B_{0, \sigma}^{\theta, r}(2)$. We further define the symbol $A(\varepsilon)=A(t, y, \eta ; \lambda, \varepsilon)$ as

$$
A(t, y, \eta ; \lambda, \varepsilon)=\omega(\eta)(-B(\eta)+Q(t, y, \eta ; \lambda, \varepsilon))
$$

for $Q(\varepsilon)=Q(t, y, \eta ; \lambda, \varepsilon)$ introduced in Lemma 6.1. The symbol $A(\varepsilon)$ has the following properties:

(6.2.1) $A(\varepsilon)$ is a $2 \times 2$ symmetric matrix;

(6.2.2) The $(j, k)$-component $a_{f k}(t, y, \eta ; \lambda, \varepsilon)$ of $A(\varepsilon)(j, k=1,2)$ has compact support in $\eta$ and satisfies the estimates

$$
\begin{aligned}
\left|\partial_{\eta}^{n} a_{j k}(t, y, \eta ; \lambda, \varepsilon)\right| \leqq & C_{N, n}(1+|\eta|)^{-N}\left\{1+\varepsilon(1+|z|)^{-(\theta-|n| \gamma)}\right\}, \\
\left|\partial_{y}^{m} \partial_{\eta}^{n} a_{j k}(t, y, \eta ; \lambda, \varepsilon)\right| \leqq \varepsilon C_{N, m, n}(1+|\eta|)^{-N}(1+|z|)^{-\mu(m, n)}, \quad|m| \geqq 1, & \quad(\mu(m, n)=\sigma+(|m|-1) \theta-|n| \gamma)
\end{aligned}
$$

for any $N$, where the constants $C_{N, n}$ and $C_{N, m, n}$ are independent of $\varepsilon$.

Next, we set

$$
\begin{aligned}
w_{+} & =\tilde{W}\left(z, D_{z} ; \lambda, \varepsilon\right) \tilde{v}_{+} \\
& =\tilde{W}\left(z, D_{z} ; \lambda, \varepsilon\right) \tilde{V}\left(D_{z}\right)\left[\tilde{U}\left(z, D_{z} ; \lambda, \varepsilon\right) \psi\left(D_{z}\right) u\right]_{+}
\end{aligned}
$$

for $\tilde{v}_{+}$of equation (5.10). Then, we have the following result.

Lemma 6.2. Let $A(t, y, \eta ; \lambda, \varepsilon)$ be defined by (6.2) and let $w_{+}$be as above. 
Then, $w_{+}$obeys the equation

$$
\partial_{t} w_{+}+i A\left(t, y, D_{y} ; \lambda, \varepsilon\right) w_{+}=i h+r_{1}(\varepsilon)
$$

with some $r_{1}(\varepsilon)$, where $h$ is defined by

$$
\begin{aligned}
h & =\tilde{T}\left(z, D_{z} ; \lambda, \varepsilon\right) \tilde{S}\left(D_{z}\right) g \\
& \left(=\tilde{T}\left(\left(z, D_{z} ; \lambda, \varepsilon\right) \tilde{S}\left(D_{z}\right)\left[\tilde{U}\left(z, D_{z} ; \lambda, \varepsilon\right) \psi\left(D_{z}\right) f\right]_{+}\right)\right.
\end{aligned}
$$

for $g$ defined by (4.6), while $r_{1}(\varepsilon)$ satisfies the estimate

$$
\left|r_{1}(\varepsilon)\right|_{0, \nu+\sigma}^{(2)} \leqq \varepsilon C_{\nu}|u|_{0, \nu}^{(1)} \quad \text { for any } \nu,
$$

if the solution $u$ to equation (4.2) belongs to $L_{2, \nu}^{(6)}$.

Equation (6.4) is derived by letting $\tilde{T}\left(z, D_{z} ; \lambda, \varepsilon\right)$ operate on both sides of equation (5.10) and by making use of Lemma 6.1. This is the desired equation and the transformation is now completed.

Now, we return to equation (4.1) with non-zero $\kappa$. We make the same transformation as (6.3) for the solution $u$ to this equation;

$$
w_{+}=\tilde{W}\left(z, D_{z} ; \lambda, \varepsilon\right) \tilde{V}\left(D_{z}\right)\left[\tilde{U}\left(z, D_{z} ; \lambda, \varepsilon\right) \psi\left(D_{z}\right) u\right]_{+} \cdot
$$

Then, by Lemma $6.2, w_{+}$satisfies the equation

$$
\partial_{t} w_{+}+i A\left(t, y, D_{y} ; \lambda, \varepsilon\right) w_{+}=i \tilde{h}+r_{2}(\varepsilon)
$$

where $\tilde{h}$ is defined by (6.5) with $f=f+i \kappa\left(I_{6}+E(x ; \varepsilon)\right) u$;

$$
\begin{aligned}
& \tilde{h}=f_{1}+i \kappa f_{2}, \\
& f_{1}=\tilde{T}\left(z, D_{z} ; \lambda, \varepsilon\right) \tilde{S}\left(D_{z}\right)\left[\tilde{U}\left(z, D_{z} ; \lambda, \varepsilon\right) \psi\left(D_{z}\right) f\right]_{+}, \\
& f_{2}=\tilde{T}\left(z, D_{z} ; \lambda, \varepsilon\right) \tilde{S}\left(D_{z}\right)\left[\tilde{U}\left(z, D_{z} ; \lambda, \varepsilon\right)\left(I_{6}+E(z ; \varepsilon)\right) \psi\left(D_{z}\right) u\right]_{+},
\end{aligned}
$$

and $r_{2}(\varepsilon)$ satisfies the estimate of the same type as (6.6). (Throughout the remainder, we denote by $r_{j}(\varepsilon), j=1,2, \cdots$, all terms satisfying the estimate of the same type as (6.6).) Since the symbols $\tilde{T}(z, \zeta ; \lambda, \varepsilon)$ and $\tilde{S}(\zeta)$ are invertible in a small neighborhood of the support of $\psi(\zeta), f_{2}$ can be decomposed into

$$
f_{2}=\tilde{S}\left(D_{z}\right)^{2} w_{+}+g_{1}+r_{3}(\varepsilon)
$$

with some $r_{3}(\varepsilon)$, where $g_{1}$ is represented in the form

$$
\begin{aligned}
g_{1}= & G\left(z, D_{z} ; \lambda, \varepsilon\right)\left[\tilde{U}\left(z, D_{z} ; \lambda, \varepsilon\right) \psi\left(D_{z}\right) u\right]_{+} \\
& +\tilde{T}\left(z, D_{z} ; \lambda, \varepsilon\right) \tilde{S}\left(D_{z}\right)\left[\tilde{U}\left(z, D_{z} ; \lambda, \varepsilon\right) E(z ; \varepsilon) \psi\left(D_{z}\right) u\right]_{+}
\end{aligned}
$$


with some $G(z, \zeta ; \lambda, \varepsilon)$ belonging to $A_{\theta, \sigma}^{\theta, r}(2)$ uniformly in $\varepsilon$. Hence, by Proposition 3.2, $g_{1} \in L_{2, \theta}^{(2)}$ and satisfies

$$
\left|g_{1}\right|_{0, \theta}^{(2)} \leqq C|u|_{0,0}^{(6)}
$$

for $C$ independent of $\varepsilon$. Summing up, we have the following result.

TheOREm 6.1. Let $u$ be the $L^{2}$-solution to equation (4.1) with $f \in L_{2, \alpha}^{(6)}$, $\alpha>\frac{1}{2}$. Define $w_{+}$by (6.7). Then, $w_{+}$satisfies the equation

$$
\partial_{t} w_{+}+i A\left(t, y, D_{y} ; \lambda, \varepsilon\right) w_{+}+\kappa \tilde{S}\left(D_{z}\right)^{2} w_{+}=i f_{1}-\kappa g_{1}+r_{4}(\varepsilon)
$$

with some $r_{4}(\varepsilon)$, where $f_{1} \in L_{2, \alpha}^{(2)}$ is defined by

$$
f_{1}=\tilde{T}\left(z, D_{z} ; \lambda, \varepsilon\right) \tilde{S}\left(D_{z}\right)\left[\tilde{U}\left(z, D_{z} ; \lambda, \varepsilon\right) \psi\left(D_{z}\right) f\right]_{+}
$$

and $g_{1}$ belongs to $L_{2, \theta}^{(2)}$ and satisfies the estimate (6.8).

(6.9) is the basic equation corresponding to (3.1) in [10]. Once this equation has derived, the proof of Theorems 1.1 and 1.2 is done exactly in the same way as [10], so we omit it.

Finally, we note that an equation similar to (6.9) can be also derived for another coordinate system $(\tilde{z}, \tilde{\zeta})$ close enough to $(z, \zeta)$.

\section{§7. Proof of Lemma 5.2}

Proof of Lemma 5.2. For notational convenience, we write $A_{+}(\zeta)$ as

$$
A_{+}(\zeta)=\left(\begin{array}{ll}
a_{1}(\zeta), & a(\zeta) \\
a(\zeta), & a_{2}(\zeta)
\end{array}\right)
$$

$\left(a_{1}(\zeta)=a_{+}^{11}(\tau, \eta), a_{2}(\zeta)=a_{+}^{22}(\tau, \eta), a(\zeta)=a_{+}^{12}(\tau, \eta)=a_{+}^{21}(\tau, \eta)\right)$.

Now, we put $S(\zeta)$ and $B(\eta)$ as follows:

$$
S(\zeta)=\left(\begin{array}{ll}
s_{1}(\zeta), & s(\zeta) \\
s(\zeta), & s_{2}(\zeta)
\end{array}\right), \quad B(\eta)=\left(\begin{array}{ll}
b_{1}(\eta), & b(\eta) \\
b(\eta), & b_{2}(\eta)
\end{array}\right) .
$$

We assume that all the components of $S(\zeta)$ and $B(\eta)$ are real and hence $S(\zeta)$ and $B(\eta)$ are symmetric matrices. (Note that the component $a(\zeta)$ is real.) Furthermore, for the moment, we assume that $S(\zeta)$ is invertible in $\mathcal{O} ; k(\zeta)=s_{1}(\zeta) s_{2}(\zeta)-s(\zeta)^{2} \neq 0$. We multiply both sides of $(5.7)$ by $S(\zeta)^{-1}$ from the right side and obtain

$$
\begin{aligned}
k(\zeta) & \left(\begin{array}{ll}
s_{1}(\zeta), & s(\zeta) \\
s(\zeta), & s_{2}(\zeta)
\end{array}\right)\left(\begin{array}{ll}
a_{1}(\zeta)-\lambda, & a(\zeta) \\
a(\zeta), & a_{2}(\zeta)-\lambda
\end{array}\right) \\
= & \left(\begin{array}{ll}
\tau-b_{1}(\eta), & -b(\eta) \\
-b(\eta), & \tau-b_{2}(\eta)
\end{array}\right)\left(\begin{array}{lr}
s_{2}(\zeta), & -s(\zeta) \\
-s(\zeta), & s_{1}(\zeta)
\end{array}\right)
\end{aligned}
$$


We take the determinant of both sides of (7.1). Then, in view of (5.5), it is natural to assume that

$$
\begin{aligned}
& k(\zeta)=D(\zeta ; \lambda)^{-1 / 2}\left(=D(\tau, \eta ; \lambda)^{-1 / 2}\right), \\
& b_{1}(\eta)+b_{2}(\eta)=\sigma_{1}(\eta ; \lambda), \\
& b_{1}(\eta) b_{2}(\eta)-b(\eta)^{2}=\sigma_{2}(\eta ; \lambda),
\end{aligned}
$$

where $D(\zeta ; \lambda)$ is defined by (5.6) and $\sigma_{\jmath}(\eta ; \lambda), j=1,2$, are introduced in decomposition (5.3). Equation (7.1) becomes four homogeneous linear equations for $\left(s_{1}(\zeta), s_{2}(\zeta), s(\zeta)\right)$. Using the relations (5.5) and (7.2) (7.4), we see after a short calculation that non-trivial solutions to these linear equations are given by

$$
\begin{aligned}
& s_{1}(\zeta)=F(\zeta)\left\{\tau+\left(a_{2}(\zeta)-\lambda\right) E(\zeta ; \lambda)-b_{1}(\eta)\right\}, \\
& s_{2}(\zeta)=F(\zeta)\left\{\tau+\left(a_{1}(\zeta)-\lambda\right) E(\zeta ; \lambda)-b_{2}(\eta)\right\}, \\
& s(\zeta)=-F(\zeta)(a(\zeta) E(\zeta ; \lambda)+b(\eta)),
\end{aligned}
$$

with any function $F(\zeta)$, where we have set $E(\zeta ; \lambda)=D(\zeta ; \lambda)^{-1 / 2}$.

We now define the smooth function $\rho(\eta)\left(\rho(0)=\tau_{0}\right), \eta \in \Omega$, as the (unique) root of the equation for $\tau$;

$$
2 \tau+\left(a_{1}(\zeta)+a_{2}(\zeta)-2 \lambda\right) E(\tau, \eta ; \lambda)=\sigma_{1}(\eta) .
$$

It is easily seen that equation (7.5) has the desired root. In fact, if we set $G(\tau, \eta)=2 \tau+\left(a_{1}(\zeta)+a_{2}(\zeta)-2 \lambda\right) E(\tau, \eta ; \lambda)-\sigma_{1}(\eta)$, then it follows from Lemma 5.1 that $G\left(\tau_{0}, 0\right)=0$. Furthermore, using Lemma 5.1 again and recalling the expression for $D(\tau, \eta ; \lambda)$ defined by $(5.6)$, we see that $(\partial / \partial \tau) G\left(\tau_{0}, 0\right)>0$. Thus, the implicit function theorem shows the existence of the desired root $\rho(\eta)$, if $\Omega$ is taken small enough.

With $\rho(\eta)$ defined above, we now determine $b_{1}(\eta), b_{2}(\eta)$ and $b(\eta)$ by

$$
\begin{aligned}
& b_{1}(\eta)=\rho(\eta)+\left(a_{2}(\rho(\eta), \eta)-\lambda\right) E(\rho(\eta), \eta ; \lambda), \\
& b_{2}(\eta)=\rho(\eta)+\left(a_{1}(\rho(\eta), \eta)-\lambda\right) E(\rho(\eta), \eta ; \lambda), \\
& b(\eta)=-a(\rho(\eta), \eta) E(\rho(\eta), \eta ; \lambda) .
\end{aligned}
$$

Here we note that we choose the neighborhood $\mathcal{O}=J \times \Omega$ so that $\rho(\eta) \in J$ for $\eta \in \Omega$. If we determine $b_{j}(\eta)(j=1,2)$ and $b(\eta)$ as above, then we see from Lemma 5.1 that $b_{1}(0)=b_{2}(0)=\tau_{0}$ and $b(0)=0$. Furthermore, (7.3) and (7.4) are satisfied. Indeed, (7.3) follows from the definition of $\rho(\eta)$ and (7.4) is verified by making use of (7.5) and (5.5).

Next, we define $t_{1}(\zeta), t_{2}(\zeta)$ and $t(\zeta)$ as 


$$
\begin{aligned}
& t_{1}(\zeta)=\left\{\tau+\left(a_{2}(\zeta)-\lambda\right) E(\zeta ; \lambda)-b_{1}(\eta)\right\} /(\tau-\rho(\eta)), \\
& t_{2}(\zeta)=\left\{\tau+\left(a_{1}(\zeta)-\lambda\right) E(\zeta ; \lambda)-b_{2}(\eta)\right\} /(\tau-\rho(\eta)), \\
& t(\zeta)=-\{a(\zeta) E(\zeta ; \lambda)+b(\eta)\} /(\tau-\rho(\eta)) .
\end{aligned}
$$

These functions are well-defined and smooth in $\mathcal{O}$, which follows from the definitions of $b_{j}(\eta)(j=1,2)$ and $b(\eta)$. Moreover, by Lemma 5.1, we have that $t_{1}\left(\xi_{0}\right)=t_{2}\left(\xi_{0}\right) \neq 0$ and $t\left(\xi_{0}\right)=0$ at $\xi_{0}=\left(\tau_{0}, 0,0\right)$. (In particular, $t\left(\xi_{0}\right)=0$ follows from (ii) in Lemma 5.1.) Hence, $H(\zeta)^{2}=t_{1}(\zeta) t_{2}(\zeta)-t(\zeta)^{2}>0$ in $\mathcal{O}$.

Now, we determine $s_{1}(\zeta), s_{2}(\zeta)$ and $s(\zeta)$ by

$$
s_{j}(\zeta)=\sqrt{E(\zeta ; \lambda)} t_{j}(\zeta) / H(\zeta), \quad(j=1,2), \quad s(\zeta)=-\sqrt{E(\zeta ; \lambda) t(\zeta) / H(\zeta)} .
$$

Then, $s_{1}(\zeta), s_{2}(\zeta)$ and $s(\zeta)$ satisfy equation (7.1). (Take $F(\zeta)=\sqrt{E(\zeta ; \lambda)} /$ $(\tau-\rho(\eta)) H(\zeta)$ as $F(\zeta)$.) And $s_{1}(\zeta) s_{2}(\zeta)-s(\zeta)^{2}=E(\zeta ; \lambda)=D(\zeta ; \lambda)^{-1 / 2}$. Hence, (7.2) is satisfied and $S(\eta)$ is invertible in $\mathcal{O}$. Thus, we can find $S(\zeta)$ and $B(\eta)$ with properties (a) and (b) and the proof is completed.

\section{§ 8. Proof of Lemma 6.1}

8.1. Preparations. Let $B(\eta), \eta \in \Omega$, be the $2 \times 2$ symmetric matrix introduced in Lemma 5.2. The $(j, j)$ and $(j, k), j \neq k$, components of $B(\eta)$ are denoted by $b_{j}(\eta)(j=1,2)$ and $b(\eta)$, respectively. In the proof of Lemma 5.2 , we have shown that

$$
b_{1}(0)=b_{2}(0)=\tau_{0}, \quad b(0)=0 .
$$

For later use, we here set

$$
b_{0}(\eta)=\frac{1}{2}\left(b_{1}(\eta)+b_{2}(\eta)\right), \quad \tilde{b}(\eta)=\frac{1}{2}\left(b_{1}(\eta)-b_{2}(\eta)\right) .
$$

We denote by $k_{j}(\eta)(j=1,2), \eta \in \Omega$, the eigenvalues of $B(\eta) ; k_{1}(\eta) \geqq$ $k_{2}(\eta)>0$. We see immediately that $k_{1}(\eta)=k_{2}(\eta)$ only for $\eta=0$ and that $k_{j}(\eta)$ are smooth in $\Omega$ except for $\eta=0$. We may choose the small neighborhood $\mathcal{O}=J \times \Omega$ of $\xi_{0}$ so that $k_{j}(\eta) \in J$ for $\eta \in \Omega$.

Let $\omega(\eta)$ be as before. We set $\omega_{2}(z, \eta)=\omega\left(M\langle z\rangle^{r} \eta\right),\langle z\rangle=\left(1+|z|^{2}\right)^{1 / 2}$, where the constant $M$ is fixed so large that the support of $\omega_{2}(z, \eta)$ in $\eta$ is contained in $\Omega$ uniformly in $z$. We further set $\omega_{1}(z, \eta)=1-\omega_{2}(z, \eta)$.

We decompose $B(\eta)$ into $B(\eta)=B_{1}(z, \eta)+B_{2}(z, \eta)$, where

$$
\begin{aligned}
& B_{1}(z, \eta)=\left(\begin{array}{cc}
b_{0}(\eta), & 0 \\
0, & b_{0}(\eta)
\end{array}\right)+\omega_{1}(z, \eta)\left(\begin{array}{lr}
\tilde{b}(\eta), & b(\eta) \\
b(\eta), & -\tilde{b}(\eta)
\end{array}\right), \\
& B_{2}(z, \eta)=\omega_{2}(z, \eta)\left(\begin{array}{lr}
\tilde{b}(\eta), & b(\eta) \\
b(\eta), & -\tilde{b}(\eta)
\end{array}\right) .
\end{aligned}
$$


We denote by $h_{\jmath}(z, \eta)(j=1,2)$ the eigenvalues of $B_{1}(z, \eta) ; h_{1} \geqq h_{2}>0$. As is easily seen, $h_{j}(z, \eta)$ are given by

$$
\begin{aligned}
& h_{1}(z, \eta)=b_{0}(\eta)+\omega_{1}(z, \eta) \sqrt{\tilde{\tilde{b}}(\eta)^{2}+b(\eta)^{2}}, \\
& h_{2}(z, \eta)=b_{0}(\eta)-\omega_{1}(z, \eta) \sqrt{\tilde{b}(\eta)^{2}+b(\eta)^{2}},
\end{aligned}
$$

and $h_{\mathrm{f}}(z, \eta)$ are smooth in $R_{z}^{3} \times \Omega$.

Lemma 8.1. Let $B_{1}(z, \eta)$ and $B_{2}(z, \eta)$ be as above. Let $h_{j}(z, \eta)(j=1,2)$ be the eigenvalues of $B_{1}(z, \eta)$. Then, the following statements hold: $B_{1}(z, \eta) \in \tilde{B}_{0,1+r}^{1, \gamma}(2 ; \Omega)$, (ii) $B_{2}(z, \eta) \in \tilde{B}_{r, 1+\gamma}^{1, \gamma}(2 ; \Omega)$, (iii) $h_{\jmath}(z, \eta) \in \tilde{B}_{0,1+\gamma}^{1, \gamma}(1 ; \Omega)$.

Proof. Since $\tilde{b}(\eta)=\mathcal{O}(|\eta|)$ and $b(\eta)=O(|\eta|)$ as $|\eta| \rightarrow 0$ by (8.1), (i) and (ii) follow at once. For the proof of (iii), we note that $c_{1}|\eta|^{2} \leqq \tilde{b}(\eta)^{2}+b(\eta)^{2}$ $\leqq c_{2}|\eta|^{2}$, which follows from the fact that the discriminant $X(\zeta)$ defined in subsection 2.2 satisfies the same estimate as above as $|\eta| \rightarrow 0$. Hence, (iii) is easily verified.

Lemma 8.2. Let $h_{j}(z, \eta)$ be as above. For given $g(z, \zeta)=g(z, \tau, \eta) \in$ $B_{\nu, o}^{\theta, r}(1 ; \mathcal{O})(\nu \geqq \theta)$, it holds that:

(i) $g\left(z, h_{1}(z, \eta), \eta\right) \in \tilde{B}_{v, \sigma}^{\theta, \gamma}(1 ; \Omega)$,

(ii) $\left\{g(z, \tau, \eta)-g\left(z, h_{1}(z, \eta), \eta\right)\right\} /\left(\tau-h_{1}(z, \eta)\right)$

A similar result is also valid for $h_{2}(z, \eta)$.

First, we should note that by our choice of $\mathcal{O}$ the two symbols above are well-defined. The proof is easily done by making use of Lemma 8.1, so we omit it.

The proof of Lemma 6.1 is done with the aid of the next lemma.

Lemma 8.3. Let $B_{1}(z, \zeta)$ be the symmetric matrix defined by (8.2). Then, for given symmetric matrix $G(z, \zeta) \in B_{v, o}^{\theta, r}(2 ; \mathcal{O})(\nu \geqq \theta)$, there exist two $2 \times 2$ symmetric matrices $P(z, \zeta)$ and $Q(z, \eta)$ such that

$$
P(z, \zeta)\left(\tau I_{2}-B_{1}(z, \zeta)\right)+\left(\tau I_{2}-B_{1}(z, \zeta)\right) P(z, \zeta)+G(z, \zeta)=Q(z, \eta) .
$$

Here $P(z, \zeta)$ and $Q(z, \eta)$ have the following properties:

(a) $P(z, \zeta)$ belongs to $B_{\nu, \sigma}^{\theta, r}(2 ; \mathcal{O})$;

(b) $Q(z, \eta)$ belongs to $\tilde{B}_{\nu, \sigma}^{\theta, \gamma}(2 ; \Omega)$.

Furthermore, if $\varepsilon^{-1} G(z, \zeta ; \varepsilon)$ belongs to $B_{\nu, \sigma}^{\theta, r}(2 ; \mathcal{O})$. uniformly in $\varepsilon$, then $\varepsilon^{-1} P(z, \zeta ; \varepsilon)$ and $\varepsilon^{-1} Q(z, \eta ; \varepsilon)$ also belong to the corresponding symbol classes uniformly in $\varepsilon$. 
8.2. Proof of Lemma 6.1. We first give the proof of Lemma 6.1, admitting the validity of Lemma 8.3 .

Proof of Lemma 6.1. We choose an integer $K$ so large that $K_{\gamma}>1$. We put formally $P(\varepsilon)$ and $Q(\varepsilon)$ as follows:

$$
P(\varepsilon)=\sum_{j=0}^{K} P_{j}(\varepsilon), \quad Q(\varepsilon)=\sum_{j=0}^{K} Q_{j}(\varepsilon) .
$$

We write $P_{-1}=Q_{-1}=I_{2}$. Here we determine $P_{j}(\varepsilon)=P_{\jmath}(z, \zeta ; \lambda, \varepsilon) Q_{\jmath}(\varepsilon)=$ $Q_{j}(z, \eta ; \lambda, \varepsilon)$ to satisfy the following equations:

$$
\begin{aligned}
& P_{0}(\varepsilon)\left(\tau I_{2}-B_{1}(z ; \eta)\right)+\left(\tau I_{2}-B_{1}(z, \eta)\right) P_{0}(\varepsilon)=-\tilde{Y}_{+}(\varepsilon)+Q_{0}(\varepsilon), \\
& P_{j}(\varepsilon)\left(\tau I_{2}-B_{1}(z ; \eta)\right)+\left(\tau I_{2}-B_{1}(z, \eta)\right) P_{j}(\varepsilon)=Q_{j}(\varepsilon) \\
& \quad+\sum_{k=-1}^{j-1} P_{k}(\varepsilon)\left(B_{2}(z, \eta)-\tilde{Y}_{+}(\varepsilon)\right) P_{j-2-k}(\varepsilon) \\
& \quad-\sum_{k=0}^{j-1} P_{k}(\varepsilon)\left(\tau I_{2}-B_{1}(z, \eta)\right) P_{j-1-k}(\varepsilon), \quad 1 \leqq j \leqq K .
\end{aligned}
$$

Furthermore, we require $P_{\jmath}(\varepsilon)$ and $Q_{\jmath}(\varepsilon)$ to have the following properties:

$(8.7 ; j) \quad P_{j}(\varepsilon)$ is symmetric and $\varepsilon^{-1} P_{j}(\varepsilon)$ belongs to $B_{j r+\theta, o}^{\theta, r}(2 ; \mathcal{O})$ uniformly in $\varepsilon, 0 \leqq j \leqq K$;

$(8.8 ; j) \quad Q_{\jmath}(\varepsilon)$ is symmetric and $\varepsilon^{-1} Q_{\jmath}(\varepsilon)$ belongs to $\tilde{B}_{j r+\theta, o}^{\theta, r}(2 ; \Omega)$ uniformly in $\varepsilon$.

By Lemma 8.3 , we can construct $P_{\jmath}(\varepsilon)$ and $Q_{\jmath}(\varepsilon)$ satisfying $(8.6 ; j) \sim(8.8 ; j)$ $(0 \leqq j \leqq K)$ inductively. If $P_{\jmath}(\varepsilon)$ and $Q_{\jmath}(\varepsilon)$ are determined to satisfy $(8.6 ; j)$ and if $P(\varepsilon)$ and $Q(\varepsilon)$ are defined as above, then we have, recalling the decomposition for $B(\eta)$, that

$$
\left(I_{2}+P(\varepsilon)\right)\left(\tau I_{2}-B(\eta)+\tilde{Y}_{+}(\varepsilon)\right)\left(I_{2}+P(\varepsilon)\right)=\tau I_{2}-B(\eta)+Q(\varepsilon)+R_{2}(\varepsilon),
$$

where $R_{2}(\varepsilon)=R_{2}(z, \zeta ; \lambda, \varepsilon)$ is expressed as

$$
\begin{aligned}
R_{2}(\varepsilon)= & \sum_{j=0}^{K} \sum_{k=j}^{K} P_{k}(\varepsilon)\left(\tau I_{2}-B_{1}(z, \eta)\right) P_{K+j-k}(\varepsilon) \\
& +\sum_{j=-1}^{K} \sum_{k=j}^{K} P_{k}(\varepsilon)\left(\tilde{Y}_{+}(\varepsilon)-B_{2}(z, \eta)\right) P_{K+j-k}(\varepsilon) .
\end{aligned}
$$

It is easy to see from $(8.7 ; j)$ and $(8.8 ; j)$ that $\varepsilon^{-} R_{2}(\varepsilon)$ belongs to $B_{\sigma, \sigma}^{\theta, r}(2 ; \mathcal{O})$ uniformly in $\varepsilon$. Furthermore, it follows from $(8.7 ; j)$ again that $P_{\jmath}(\varepsilon)=O(\varepsilon)$ 
and hence $I_{2}+P(\varepsilon)$ is invertible in $\mathcal{O}$ for $\varepsilon$ small enough. Thus, we can construct $P(\varepsilon)$ and $Q(\varepsilon)$ with the desired properties (a) and (b) and the proof is now completed.

8.3. Proof of Lemma 8.3. Finally we shall prove Lemma 8.3.

Proof of Lemma 8.3. For given $G(z, \zeta)=G(z, \tau, \eta)$, we write

$$
G(z, \zeta)=\left(\begin{array}{ll}
2 g_{1}(z, \zeta), & g(z, \zeta) \\
g(z, \zeta), & 2 g_{2}(z, \zeta)
\end{array}\right)
$$

(For brevity, we assume that $g(z, \zeta)$ is real-valued.) We use the following notations for given $k(z, \eta)$ and $h(z, \eta)$ :

$$
\begin{aligned}
& K(z, \zeta ; k)=\frac{1}{\tau-k(z, \eta)}\{g(z, \tau, \eta)-g(z, k(z, \eta), \eta)\} \\
& H(z, \zeta ; h, k)=\frac{1}{\tau-h(z, \eta)}\{K(z, \tau, \eta ; k)-K(z, h(z, \eta), \eta ; k)\} .
\end{aligned}
$$

(We assume that the symbols above are well-defined.) If $k(z, \eta)$ and $h(z, \eta)$ belong to $\tilde{B}_{0,1+r}^{1, r}(1 ; \Omega)$, it then follows from Lemma 8.2 that $K(z, \zeta ; k)$ and $H(z, \zeta ; h, k)$ belong to $B_{\nu, \sigma}^{\theta, r}(1 ; \mathcal{O})$. In a similar way, we introduce the notations $K_{j}(z, \zeta ; k)$ and $H_{j}(z, \zeta ; h, k)$ for $g_{j}(z, \tau, \eta)(j=1,2)$.

Now, we put $P(z, \zeta)$ and $Q(z, \eta)$ as follows:

$$
P(z, \zeta)=\left(\begin{array}{ll}
p_{1}(z, \zeta), & p(z, \zeta) \\
p(z, \zeta), & p_{2}(z, \zeta)
\end{array}\right), \quad Q(z, \eta)=\left(\begin{array}{ll}
2 q_{1}(z, \eta), & q(z, \eta) \\
q(z, \eta), & 2 q_{2}(z, \eta)
\end{array}\right),
$$

We assume that the components of $P(z, \zeta)$ and $Q(z, \eta)$ are real-valued and hence $P(z, \zeta)$ and $Q(z, \eta)$ are symmetric matrices. Furthermore, we put the components of $B_{1}(z, \eta)$ as follows:

$$
\begin{aligned}
& c_{1}(z, \eta)=b_{0}(\eta)+\omega_{1}(z, \eta) \tilde{b}(\eta) \\
& c_{2}(z, \eta)=b_{0}(\eta)-\omega_{1}(z, \eta) \tilde{b}(\eta) \\
& c(z, \eta)=\omega_{1}(z, \eta) b(\eta), \quad\left(c_{1}+c_{2}=2 b_{0}\right) .
\end{aligned}
$$

Then, (8.5) becomes linear equations for $\left(p_{1}, p_{2}, p\right)$;

$$
\begin{aligned}
& \left(\tau-c_{1}\right) p_{1}-c p+g_{1}=q_{1}, \\
& \left(\tau-c_{2}\right) p_{2}-c p+g_{2}=q_{2}, \\
& 2\left(\tau-b_{0}\right) p-c p_{1}-c p_{2}+g=q .
\end{aligned}
$$

We eliminate $p_{1}$ and $p_{2}$ from the above equations to obtain 


$$
2\left(\tau-b_{0}\right)\left\{\tau^{2}-\left(c_{1}+c_{2}\right) \tau+c_{1} c_{2}-c^{2}\right\} p=Z(z, \zeta)
$$

where

$$
\begin{aligned}
Z(z, \zeta)= & Z(z, \tau, \eta) \\
= & c\left\{\left(\tau-c_{2}\right)\left(q_{1}-g_{1}\right)+\left(\tau-c_{1}\right)\left(q_{2}-g_{2}\right)\right\} \\
& \quad+\left(\tau-c_{1}\right)\left(\tau-c_{2}\right)(q-g) .
\end{aligned}
$$

With the eigenvalues $h_{j}(z, \eta)(j=1,2)$ of $B_{1}(z, \eta)$ (defined by (8.4)), (8.10) can be rewritten as

$$
2\left(\tau-b_{0}\right)\left(\tau-h_{1}\right)\left(\tau-h_{2}\right) p=Z(z, \zeta)
$$

As the first step, we require $Z(z, \zeta)$ to satisfy $Z\left(z, b_{0}(\eta), \eta\right)=0$. This implies that

$$
c q_{1}=c\left\{g_{1}\left(b_{0}\right)+q_{2}-g_{2}\left(b_{0}\right)\right\}+\left(c_{1}-b_{0}\right)\left(q-g\left(b_{0}\right)\right),
$$

where we have set $g\left(b_{0}\right)=g\left(z, b_{0}(\eta), \eta\right)$ (similarly for $\left.g_{j}\left(b_{0}\right), j=1,2\right)$. (By our choice of the neighborhood $\mathcal{O}=J \times \Omega$, these symbols are well-defined.) We insert the expression (8.12) for $c q_{1}$ into (8.11) and obtain

$$
2\left(\tau-h_{1}\right)\left(\tau-h_{2}\right) p=Z_{1}(z, \zeta)
$$

where

$$
\begin{aligned}
Z_{1}(z, \zeta)= & Z_{1}(z, \tau, \eta) \\
= & 2 c\left(q_{2}-g_{2}\left(b_{0}\right)\right)+\left(\tau-c_{2}\right)\left(q-g\left(b_{0}\right)\right) \\
& -c\left\{\left(\tau-c_{2}\right) K_{1}\left(z, \zeta ; b_{0}\right)+\left(\tau-c_{1}\right) K_{2}\left(z, \zeta ; b_{0}\right)\right\} \\
& -\left(\tau-c_{1}\right)\left(\tau-c_{2}\right) K\left(z, \zeta ; b_{0}\right) .
\end{aligned}
$$

As in the first step, we set $Z_{1}\left(z, h_{1}(z, \eta), \eta\right)=0$ and obtain, using the relation $h_{1}^{2}-\left(c_{1}+c_{2}\right) h_{1}+c_{1} c_{2}=c^{2}$, the expression for $2 c q_{2}$;

$$
\begin{aligned}
2 c q_{2}= & 2 c g_{2}\left(b_{0}\right)-\left(h_{1}-c_{2}\right)\left(q-g\left(b_{0}\right)\right) \\
& +c\left\{\left(h_{1}-c_{2}\right) K_{1}\left(h_{1} ; b_{0}\right)+\left(h_{1}-c_{1}\right) K_{2}\left(h_{1} ; b_{0}\right)+c K\left(h_{1} ; b_{0}\right)\right\},
\end{aligned}
$$

where $K\left(h_{1} ; b_{0}\right)=K\left(z, h_{1}(z, \eta), \eta ; b_{0}\right)$ (similarly for $K_{\jmath}\left(h_{1} ; b_{0}\right), j=1,2$ ). We insert (8.14) into (8.13) and obtain, using the relation $\left(\tau-h_{1}\right)\left(\tau-h_{2}\right)=\tau^{2}$ $-\left(c_{1}+c_{2}\right) \tau+c_{1} c_{2}-c^{2}$, that

$$
2\left(\tau-h_{2}\right) p=Z_{2}(z, \zeta)
$$


where

$$
\begin{aligned}
Z_{2}(z, \zeta)= & Z_{2}(z, \tau, \eta) \\
= & q-g\left(b_{0}\right)-\left(\tau-h_{2}\right) K\left(z, \zeta ; b_{0}\right)-c\left\{K_{1}\left(h_{1} ; b_{0}\right)+K_{2}\left(h_{1} ; b_{0}\right)\right. \\
& +c H\left(z, \zeta ; h_{1}, b_{0}\right)+\left(\tau-c_{2}\right) H_{1}\left(z, \zeta ; h_{1}, b_{0}\right) \\
& \left.\quad+\left(\tau-c_{1}\right) H_{2}\left(z, \zeta ; h_{1}, b_{0}\right)\right\} .
\end{aligned}
$$

Finally, setting $Z_{2}\left(z, h_{2}(z, \eta), \eta\right)=0$, we can determine $q$ in the form

$$
q=g\left(b_{0}\right)+c S(z, \eta)
$$

with some $S(z, \eta) \in \tilde{B}_{\nu, \sigma}^{\theta, \gamma}(1 ; \Omega)$ and hence $q \in \tilde{B}_{\nu, \sigma}^{\theta, \gamma}(1 ; \Omega)$. Furthermore, combining (8.15) with (8.14) and (8.12), we can determine $q_{1}$ and $q_{2}$ with the desired properties. Thus, $p=p(z, \zeta)$ can be also determined and it belongs to $B_{\nu, \sigma}^{\theta, r}(1 ; \mathcal{O})$.

Next, we use the relation (8.9.1) to determine $p_{1}$. To do this, we have to show that

$$
c p\left(z, c_{1}(z, \eta), \eta\right)+q_{1}(z, \eta)-g_{1}\left(z, c_{1}(z, \eta), \eta\right)=0 .
$$

However, this relation is readily verified by making use of (8.10). Similarly, $p_{2}$ can be also determined by using the relation (8.9.2). On the other hand, we can easily show that (8.9.3) is satisfied for $\left(p_{1}, p_{2}, p\right)$ satisfying (8.9.1), (8.9.2) and (8.10). Thus, we can construct $P(z, \eta)$ and $Q(z, \eta)$ with the desired properties and the proof is now completed.

\section{REFERENCES}

[1] G. S. S. Avila, Spectral resolution of differential operators associated with symmetric hyperbolic systems, Applicable Anal., 1 (1972), 283-299.

[2] R. Courant and D. Hilbert, Methods of Mathematical Physics, 2, New York, Interscience Publishers, 1962.

[ 3 ] L. Hörmander, Pseudo-differential operators and hypoelliptic equations, Proc. Symposium of Singular Integrals, Amer. Math. Soc., 10 (1967), 138-183.

[4] H. Kumano-go, Pseudo-differential operators, Tokyo, Iwanami Publishers, 1974 (in Japanese).

[ 5 ] J. A. La Vita, J. R. Schulenberger and C. H. Wilcox, The scattering theory of Lax and Phillips and wave propagation problems of classical physics, Applicable Anal., 3 (1973), 57-77.

[6] M. Murata, Rate of decay of local energy and wave operators for symmetric systems, J. Math. Soc. Japan, 31 (1979), 451-480.

[ 7 ] M. Schechter, Scattering theory for elliptic systems, J. Math. Soc. Japan, 28 (1976), 71-79.

[ 8 ] J. R. Schulenberger, A local compactness theorem for wave propagation problems of classical physics, Indiana Univ. Math. J., 22 (1972), 429-433. 
[9] H. Tamura, Spectral analysis for perturbed Laplace operators in cylindrical domains, Integral Equations and Operator Theory, 2 (1979), 69-115.

[10] - The principle of limiting absorption for uniformly propagative systems with perturbations of long-range class, Nagoya Math. J., 82 (1981), 141-174.

Department of Engineering Mathematics

Faculty of Engineering

Nagoya University

Furo-cho, Chikusa-ku, Nagoya

464, Japan 